\title{
Boundary element-free method for fracture analysis of 2-D anisotropic piezoelectric solids
}

\author{
K. M. Liew*, ${ }^{*}$, Yuzhou Sun and S. Kitipornchai \\ Department of Building and Construction, City University of Hong Kong, Tat Chee Avenue, \\ Kowloon, Hong Kong SAR
}

\begin{abstract}
SUMMARY
This paper considers a 2-D fracture analysis of anisotropic piezoelectric solids by a boundary elementfree method. A traction boundary integral equation (BIE) that only involves the singular terms of order $1 / r$ is first derived using integration by parts. New variables, namely, the tangential derivative of the extended displacement (the extended displacement density) for the general boundary and the tangential derivative of the extended crack opening displacement (the extended displacement dislocation density), are introduced to the equation so that solution to curved crack problems is possible. This resulted equation can be directly applied to general boundary and crack surface, and no separate treatments are necessary for the upper and lower surfaces of the crack. The extended displacement dislocation densities on the crack surface are expressed as the product of the characteristic terms and unknown weight functions, and the unknown weight functions are modelled using the moving least-squares (MLS) approximation. The numerical scheme of the boundary element-free method is established, and an effective numerical procedure is adopted to evaluate the singular integrals. The extended 'stress intensity factors' (SIFs) are computed for some selected example problems that contain straight or curved cracks, and good numerical results are obtained. Copyright (c) 2006 John Wiley \& Sons, Ltd.
\end{abstract}

Received 26 July 2005; Revised 19 April 2006; Accepted 27 April 2006

KEY WORDS: anisotropic piezoelectric material; crack; integration by parts; boundary integral equation; moving least-squares approximation; boundary element-free method

\section{INTRODUCTION}

Piezoelectric materials are widely used in sensors, transducers, actuators, and adaptive structures. The basic characteristic of piezoelectric materials is the coupling between the electric effect and mechanical deformation. The piezoelectric effect always appears in crystals that are anisotropic.

\footnotetext{
*Correspondence to: K. M. Liew, Department of Building and Construction, City University of Hong Kong, Tat Chee Avenue, Kowloon, Hong Kong SAR.

†E-mail: kmliew@ cityu.edu.hk
}

Contract/grant sponsor: CityU SRG; contract/grant number: 7001961 
Piezoelectric materials are brittle, and due to manufacturing process and complex electromechanical loads, are likely to develop cracks. Thus, anisotropic piezoelectric fracture analysis is important in the practical application of piezoelectric materials, and some significant analytical studies have been carried out [1-4].

The boundary element method (BEM) shows particular merit for crack problems. However, the traditional boundary integral equation (BIE) cannot be directly used to cracked bodies because of the geometrical overlapping of the upper and lower surfaces of the crack [5, 6]. Different approaches have been proposed to overcome this difficulty. Current popular techniques mainly include the hyper-singular traction integral equation method [7,8] and the dual BEM [9, 10]. In these two methods, kernels of the equation involve hyper-singular term and the treatment of hyper-singular integrals is always arduous. The singularity of integral kernels can be reduced if the technique of integration by parts is employed. Some research works in this area have already been carried out [5,11-13]. Within the scope of BEM for the fracture analysis of anisotropic piezoelectric materials, some studies [14-19] have been published in the past, but most of these works are based on the displacement BIE and are only suitable for simple problems. Recently, Pan [20] has proposed a single-domain BEM formulation and Garcia et al. [21] have presented a mixed boundary element for 2-D anisotropic piezoelectric fracture problems. In a similar way to the dual BEM, Pan [20] and Garcia et al. [21] used the extended displacement and extended hyper-singular traction integral equations on the outside boundary and on the crack surface, respectively. However, unlike the original dual BEM, only one side of the crack surfaces needs to be discretized in their methods [20,21].

In this paper, the technique of integration by parts is used and an extended traction BIE is derived for cracked 2-D anisotropic piezoelectric bodies. The kernels of the obtained integral equation involve only a singularity of order $1 / r$, so that the use of hyper-singular integrals is avoided. In comparison to the dual BEM, a distinct characteristic of the new BIE is that it can be directly applied to general boundary and crack surface, and the upper and lower surfaces of the crack do not have to be considered separately. Moreover, the tangential derivative of the extended displacement and tangential derivative of extended crack opening displacement (extended displacement dislocation densities) are introduced into the equation, and the application of the equation to curved cracks becomes convenient. Although the integration has to be performed to calculate the extended displacement and extended crack opening displacement, the extended 'stress intensity factors' (SIFs) can be easily evaluated from the extended displacement dislocation density. So the new equation is effective for the fracture mechanics analysis.

In order to study these straight and curved crack problems, a boundary element-free method is established. Based on the flexibility of the moving least-squares (MLS) approximation, the extended displacement dislocation densities on the crack surface are expressed as the product of the characteristic terms and unknown weight functions, and the unknown weight functions are modelled using the MLS approximation. In deriving the MLS shape function, an orthogonal basis function set [22-25] is used to avoid ill conditioning and improve the accuracy of results. Because the MLS shape functions are unknown in a closed form, the singular integrals must be evaluated using a numerical scheme. An efficient numerical procedure developed by Torino [26] is employed here to evaluate the Cauchy singular integrals.

Several example problems involve straight and circular-arc cracks are chosen to test the proposed method of solution. Very accurate numerical results are obtained, and the method is proven to be simple and effective. 


\section{BASIC EQUATIONS}

Under the condition of static deformation, the basic equations that govern the elastic and electric fields of a linear piezoelectric material can be summarized in the following $[1-4,20]$.

\subsection{Equilibrium equations}

The equilibrium equations are

$$
\begin{array}{r}
\sigma_{j i, i}+F_{i}=0 \\
D_{i, i}-Q=0
\end{array}
$$

where $\sigma_{j i}$ is the stress, $F_{i}$ is the body force per unit volume, $D_{i}$ is the electric displacement and $Q$ is the intrinsic electric charge per unit volume.

\subsection{Constitutive equations}

The constitutive equations are

$$
\begin{aligned}
\sigma_{i j} & =C_{i j l m} \gamma_{l m}-\mathrm{e}_{k j i} E_{k} \\
D_{i} & =\mathrm{e}_{i j k} \gamma_{j k}+\varepsilon_{i j} E_{j}
\end{aligned}
$$

where $\gamma_{l m}$ is the strain, $E_{k}$ is the electric field, $C_{i j l m}$ is the elastic modulus as measured in a constant electric field, $\mathrm{e}_{i j k}$ are the piezoelectric coefficients, and $\varepsilon_{i j}$ are the dielectric constants as measured at a constant strain.

\subsection{Strain and electric fields}

The strain and electric fields are

$$
\begin{aligned}
\gamma_{i j} & =\frac{1}{2}\left(u_{i, j}+u_{j, i}\right) \\
E_{i} & =-\phi_{i, i}
\end{aligned}
$$

where $u_{i}$ is the elastic displacement and $\phi$ is the electric potential.

In these equations, the lowercase subscripts take on a range of $1-2$, and the subscript comma denotes partial differentiation.

Using the notation that was introduced by Barnett and Lothe [1], the elastic displacement and electric potential, the elastic strain and electric field, the stress and electric displacement, and the elastic and electric moduli can be grouped in the following extended expressions:

$$
\begin{gathered}
u_{I}= \begin{cases}u_{i}, & I=1,2 \\
\phi, & I=3\end{cases} \\
\gamma_{I j}= \begin{cases}\gamma_{i j}, & I=1,2 \\
-E_{j}, & I=3\end{cases}
\end{gathered}
$$




$$
\begin{gathered}
\sigma_{i J}= \begin{cases}\sigma_{i j}, & I=1,2 \\
D_{i}, & I=3\end{cases} \\
C_{i J K l}= \begin{cases}C_{i j k l}, & J, K=1,2 \\
\mathrm{e}_{l i j}, & J=1,2 ; K=3 \\
\mathrm{e}_{i k l}, & J=3 ; K=1,2 \\
-\varepsilon_{i l}, & J, K=3\end{cases}
\end{gathered}
$$

In this definition, the lowercase subscripts take on the range of 1-2, and the uppercase subscripts take on the range of 1-3. The equilibrium equations in (1) can then be grouped as

$$
\sigma_{i J_{, i}}+F_{J}=0
$$

with $F_{J}$ being defined as

$$
F_{J}= \begin{cases}F_{j}, & J=1,2 \\ -Q, & J=3\end{cases}
$$

The constitutive equations can be unified into a single equation, i.e.

$$
\sigma_{i J}=C_{i J L m} \frac{\partial u_{L}}{\partial x_{m}}
$$

\section{THE EXTENDED GREEN'S FUNCTIONS}

Define the complex variables $z_{0 M}$ and $z_{M}$ on the complex plane as

$$
\begin{gathered}
z_{0 M}=x_{01}+\mu_{M} x_{02} \\
z_{M}=x_{1}+\mu_{M} x_{2}
\end{gathered}
$$

where $\mu_{M}$ are the three distinct complex roots with positive imaginary parts of the following characteristic equation:

$$
\left|C_{1 I J 1}+\left(C_{1 I J 2}+C_{2 I J 1}\right) \mu+C_{2 I J 2} \mu^{2}\right|=0
$$

and the extended Green's functions $U_{K L}$ and $T_{K L}$ can be explained as the $L$ th extended displacement (the elastic displacement or electric potential) and the extended traction at field point $z\left(x_{1}, x_{2}\right)$ that is caused by a unit point force (for $K=1,2$ ) along the $K$ th direction or a unit electric charge (for $K=3$ ) at the source point $z_{0}\left(x_{01}, x_{02}\right)$.

These extended fundamental solutions have been analytically obtained by Pan [20]. The extended displacement fundamental solution $U_{K L}$ can be expressed as

$$
U_{K L}\left(z_{0}, z\right)=-\frac{1}{\pi} \operatorname{Re}\left[\sum_{J=1}^{3} G_{L J} H_{J K} \ln \left(z_{J}-z_{0 J}\right)\right]
$$


where $G_{L J}$ is the component of the eigenmatrix $\mathbf{G}$ which column can be obtained for each characteristic root $\mu_{M}$ from the following equation:

$$
\left|C_{1 I J 1}+\left(C_{1 I J 2}+C_{2 I J 1}\right) \mu_{M}+C_{2 I J 2} \mu_{M}^{2}\right| G_{J M}=0
$$

For convenience, we write the traction fundamental solution $T_{K L}$ as

$$
T_{K L}\left(z_{0}, z\right)=-D_{K j L}\left(z_{0}, z\right) \cdot n_{j}(z)
$$

with

$$
\begin{aligned}
& D_{K 1 L}\left(z_{0}, z\right)=-\frac{1}{\pi} \operatorname{Re}\left[\sum_{J=1}^{3} E_{L J} H_{J K} \frac{\mu_{J}}{z_{j}-z_{0 j}}\right] \\
& D_{K 2 L}\left(z_{0}, z\right)=\frac{1}{\pi} \operatorname{Re}\left[\sum_{J=1}^{3} E_{L J} H_{J K} \frac{1}{z_{j}-z_{0 j}}\right]
\end{aligned}
$$

In the above equations, $n_{j}(z)$ are the components of the unit outward normal at the field point, and $\operatorname{Re}[]$ denotes the real part of the complex number between the brackets. Matrix $\mathbf{E}$ can be obtained by

$$
E_{I M}=\sum_{J=1}^{3}\left(C_{1 J I 2}+\mu_{M} C_{2 I J 2}\right) G_{J M}
$$

and matrix $\mathbf{H}$ from

$$
\mathbf{H}=\mathbf{G}^{-1}\left(\mathbf{Y}^{-1}+\overline{\mathbf{Y}}^{-1}\right)^{-1}
$$

with

$$
\mathbf{Y}=\mathrm{iGE}^{-1}, \quad \mathrm{i}=\sqrt{-1}
$$

\section{BOUNDARY INTEGRAL EQUATION}

From the fundamental solutions $U_{K L}\left(z_{0}, z\right)$ and $T_{K L}\left(z_{0}, z\right)$, the extended displacement integral formulation for 2-D cracked piezoelectric bodies can be derived using the Betti-type reciprocity principle

$$
\begin{aligned}
u_{I}\left(z_{0}\right)= & \int_{S} U_{I J}\left(z_{0}, z\right) t_{J}(z) \mathrm{d} s(z)-\int_{S} T_{I J}\left(z_{0}, z\right) u_{J}(z) \mathrm{d} s(z) \\
& +\int_{\Gamma} U_{I J}\left(z_{0}, z\right) \sum t_{J}(z) \mathrm{d} s(z)-\int_{\Gamma} T_{I J}\left(z_{0}, z\right) \Delta u_{J}(z) \mathrm{d} s(z)
\end{aligned}
$$

where $s(z)$ is the arc length along the boundary $S$ or crack surface $\Gamma ; \sum t_{J}(z)=t_{J}^{+}(z)+t_{J}^{-}(z)$ is the sum of the extended tractions that act on the upper and lower crack surfaces; $\Delta u_{J}(z)$ is the 
extended crack opening displacement defined as

$$
\Delta u_{J}(z)=\left(\begin{array}{c}
u_{1}^{+}(z)-u_{1}^{-}(z) \\
u_{2}^{+}(z)-u_{2}^{-}(z) \\
\phi^{+}(z)-\phi^{-}(z)
\end{array}\right)
$$

Differentiating $u_{1}\left(z_{0}\right)$ with respect to $x_{0 k}$, we have

$$
\begin{aligned}
\frac{\partial u_{I}\left(z_{0}\right)}{\partial x_{0 k}}= & \int_{S} \frac{\partial U_{I J}\left(z_{0}, z\right)}{\partial x_{0 k}} t_{J}(z) \mathrm{d} s(z)-\int_{S} \frac{\partial T_{I J}\left(z_{0}, z\right)}{\partial x_{0 k}} u_{J}(z) \mathrm{d} s(z) \\
& +\int_{\Gamma} \frac{\partial U_{I J}\left(z_{0}, z\right)}{\partial x_{0 k}} \sum t_{J}(z) \mathrm{d} s(z)-\int_{\Gamma} \frac{\partial T_{I J}\left(z_{0}, z\right)}{\partial x_{0 k}} \Delta u_{J}(z) \mathrm{d} s(z)
\end{aligned}
$$

The following relationship can be easily seen from (13):

$$
\frac{\partial U_{I J}\left(z_{0}, z\right)}{\partial x_{0 k}}=-\frac{\partial U_{I J}\left(z_{0}, z\right)}{\partial x_{k}}=-U_{I J, k}\left(z_{0}, z\right)
$$

The substitution of $D_{I l J}\left(z_{0}, z\right)$ into equilibrium equation (8) gives

$$
\frac{\partial D_{I 1 J}\left(z_{0}, z\right)}{\partial x_{1}}=-\frac{\partial D_{I 2 J}\left(z_{0}, z\right)}{\partial x_{2}} \text { for } z \neq z_{0}
$$

Using (24), we can obtain an important relationship

$$
\begin{aligned}
\frac{\partial T_{I J}\left(z_{0}, z\right)}{\partial x_{01}} & =-\left(n_{1} \frac{\partial D_{I 1 J}\left(z_{0}, z\right)}{\partial x_{01}}+n_{2} \frac{\partial D_{I 2 J}\left(z_{0}, z\right)}{\partial x_{01}}\right) \\
& =n_{1} \frac{\partial D_{I 1 J}\left(z_{0}, z\right)}{\partial x_{1}}+n_{2} \frac{\partial D_{I 2 J}\left(z_{0}, z\right)}{\partial x_{1}} \\
& =-\left(-n_{2} \frac{\partial}{\partial x_{1}}+n_{1} \frac{\partial}{\partial x_{2}}\right) D_{I 2 J}\left(z_{0}, z\right)=-\frac{\partial D_{I 2 J}\left(z_{0}, z\right)}{\partial s(z)}
\end{aligned}
$$

and the last step of (25) results from the definition of the tangential derivative

$$
\partial f / \partial s=n_{1}\left(\partial f / \partial x_{2}\right)-n_{2}\left(\partial f / \partial x_{1}\right)
$$

Similarly, we can also obtain

$$
\frac{\partial T_{I J}\left(z_{0}, z\right)}{\partial x_{02}}=\frac{\partial D_{I 1 J}\left(z_{0}, z\right)}{\partial s(z)}
$$

Formulas (26) and (27) can be written in a more compact form as

$$
\frac{\partial T_{I J}\left(\mathrm{z}_{0}, z\right)}{\partial x_{0 k}}=-\alpha_{k l} \frac{\partial D_{I l J}\left(\mathrm{z}_{0}, z\right)}{\partial s(z)}
$$

where $\alpha_{i j}=\delta_{1 i} \delta_{2 j}-\delta_{2 i} \delta_{1 j}$ is a 2 -D permutation tensor (i.e. $\alpha_{11}=\alpha_{22}=0, \alpha_{12}=-\alpha_{21}=1$ ). 
Substituting Equations (23) and (28) into (22) and inserting the expression for $\partial u_{I}\left(z_{0}\right) / \partial x_{0 k}$ into Equation (10) yield

$$
\begin{aligned}
\sigma_{l M}\left(z_{0}\right)= & -\int_{S} c_{l M I k} U_{I J, k}\left(z_{0}, z\right) t_{J}(z) \mathrm{d} s(z)+\int_{S} \frac{\partial W_{J l M}\left(z_{0}, z\right)}{\partial s(z)} u_{J}(z) \mathrm{d} s(z) \\
& -\int_{\Gamma} c_{l M I k} U_{I J, k}\left(z_{0}, z\right) \sum t_{J}(z) \mathrm{d} s(z)+\int_{\Gamma} \frac{\partial W_{J l M}\left(z_{0}, z\right)}{\partial s(z)} \Delta u_{J}(z) \mathrm{d} s(z)
\end{aligned}
$$

where $W_{J l M}\left(z_{0}, z\right)=c_{l M I k} \alpha_{k n} D_{I n J}\left(z_{0}, z\right)$.

Since the extended Green's displacement $U_{K L}\left(z_{0}, z\right)$ is a symmetric tensor [27], for the first integral kernel of (29) we have

$$
c_{l M I k} U_{J I, k}\left(z_{0}, z\right)=c_{l M I k} U_{I J, k}\left(z_{0}, z\right)=-D_{J l M}
$$

Applying integration by parts to the second integral of (29) and noting that $\left[W_{J l M} u_{J}\right]$ vanishes around a closed boundary $S$ because it is a single-value function and that $\left[W_{J l M} u_{J}\right]$ vanishes on both tips of any crack $\Gamma$, we thus obtain

$$
\begin{aligned}
\sigma_{l M}\left(z_{0}\right)= & \int_{S} D_{J l M}\left(z_{0}, z\right) t_{J}(z) \mathrm{d} s(z)-\int_{S} W_{J l M}\left(z_{0}, z\right) \frac{\partial u_{J}(z)}{\partial s(z)} \mathrm{d} s(z) \\
& \int_{\Gamma} D_{J l M}\left(z_{0}, z\right) \sum t_{J}(z) \mathrm{d} s(z)-\int_{\Gamma} W_{J l M}\left(z_{0}, z\right) \frac{\partial \Delta u_{J}(z)}{\partial s(z)} \mathrm{d} s(z)
\end{aligned}
$$

Assuming that point $z_{0}^{\prime}$ is a point on the smooth boundary $S$ or crack surface $\Gamma$, and that $n_{1}\left(z_{0}^{\prime}\right)$, $n_{2}\left(z_{0}^{\prime}\right)$ are the unit outward normal components of point $z_{0}^{\prime}$, the traction at the point $z_{0}^{\prime}$ can be expressed as

$$
t_{M}\left(z_{0}^{\prime}\right)=\lim _{z_{0} \rightarrow z_{0}^{\prime}}\left[\sigma_{l M}\left(z_{0}\right) n_{l}\left(z_{0}^{\prime}\right)\right]
$$

After substituting (31) into (32), the limiting operation gives

$$
\begin{aligned}
& n_{l}\left(z_{0}\right) \int_{S} D_{J l M}\left(z_{0}, z\right) t_{J}(z) \mathrm{d} s(z)-n_{l}\left(z_{0}\right) \int_{S} W_{J l M}\left(z_{0}, z\right) \frac{\partial u_{J}(z)}{\partial s(z)} \mathrm{d} s(z) \\
& n_{l}\left(z_{0}\right) \int_{\Gamma} D_{J l M}\left(z_{0}, z\right) \sum t_{J}(z) \mathrm{d} s(z)-n_{l}\left(z_{0}\right) \int_{\Gamma} W_{J l M}\left(z_{0}, z\right) \frac{\partial \Delta u_{J}(z)}{\partial s(z)} \mathrm{d} s(z) \\
& = \begin{cases}\frac{1}{2} t_{M}\left(z_{0}\right), & z_{0} \in s \\
\frac{1}{2}\left(t_{M}\left(z_{0}^{+}\right)-t_{M}\left(z_{0}^{-}\right)\right), & z_{0} \in \Gamma\end{cases}
\end{aligned}
$$

where $t_{M}\left(z_{0}^{+}\right)-t_{M}\left(z_{0}^{-}\right)$is the difference of the extended tractions between the upper and lower crack surfaces.

A distinct characteristic is that the integral kernels of BIE (33) do not involve hyper-singular terms, which will be very helpful for the numerical computation. Integration by parts produces 
the new variables and $\partial u_{I} / \partial s$ and $\partial \Delta u_{I} / \partial s$, which present the extended displacement densities and displacement dislocation densities, respectively. The extended SIFs can be directly evaluated from $\partial \Delta u_{I} / \partial s$. However, to obtain the extended displacement and crack opening displacement, the integrals must be performed along the boundary or crack surface. This may be a disadvantage of the current method.

All of the crack boundary conditions (both upper and lower) have been incorporated into Equation (33), thus the upper and lower surfaces of the crack do not have to be considered separately when (33) is used. In contrast to the dual BEM [10,20,21], (33) can be used directly in the proposed method for the general boundary and crack surface, and no displacement BIE is needed.

The numerical framework of the BEM that is based on (33) can be established as in References $[28,29]$ for anisotropic elastic fracture problems. In the following sections, we combine the MLS approximation with (33) to present a boundary element-free method.

\section{MOVING LEAST-SQUARES APPROXIMATION FOR THE UNKNOWNS}

In both the BEM and the FEM for linear elastic fracture analysis, the modelling of the crack-tip field is a complex and vital task. In some boundary numerical methods [30-33], the crack opening displacement is simply expressed as

$$
\Delta u_{i}(x)=\sqrt{\left(\frac{c_{1}-c_{2}}{2}\right)^{2}-\left(x-\frac{c_{1}+c_{2}}{2}\right)^{2}} F_{i}(x)
$$

where $c_{1}$ and $c_{2}$ are the co-ordinates of the two tips of a straight crack that is located on the $x$-axis. This simple approximation can give good results for general straight cracks.

If the curvilinear co-ordinate is used on a curved crack surface, then the crack opening displacement can similarly be expressed as

$$
\Delta u_{i}(s)=\sqrt{\left(\frac{s_{1}-s_{2}}{2}\right)^{2}-\left(s-\frac{s_{1}+s_{2}}{2}\right)^{2}} F_{i}(s)
$$

where $s_{1}$ and $s_{2}$ are the arc co-ordinates of the two crack tips. This approach was used by Noda and Oda [34] and Wang et al. [35], and good results were obtained for circular-arc crack and curved cracks that emanate from the adjacent holes.

Inspired by these methods, we attempt in this work to find the solutions of the extended dislocation densities in the form of

$$
\frac{\partial \Delta u_{I}}{\partial s}(s)=\frac{F_{I}(s)}{\sqrt{\left(\frac{s_{1}-s_{2}}{2}\right)^{2}-\left(s-\frac{s_{1}+s_{2}}{2}\right)^{2}}}
$$

In previous research works [30-35], the unknown variables $F_{i}(x)$ and $F_{i}(s)$ in (34) and (35) were interpolated with the power series. However, the ability of the power series interpolation is very limited. Particularly, the local numerical computation associated with the power series interpolation is always very complicated. The MLS approximation is a new flexible technique, 
and possess characteristic of both the local and global interpolation. Here, we employ the MLS approximation to model of $F_{I}(s)$ of (36), thus the local numerical computation can be easily performed.

The MLS approximation is summarily described in the following, and the related details can be gleaned from a review paper by Belytschko et al. [36]. In the MLS approximation, the trail function is

$$
F^{h}(\mathbf{x})=\sum_{i=1}^{m} p_{i}(\mathbf{x}) a_{i}(\mathbf{x})=\mathbf{p}^{\mathrm{T}}(\mathbf{x}) \mathbf{a}(\mathbf{x})
$$

where $p_{i}(\mathbf{x}), i=1,2, \ldots, m$ is the monomial basis function, $m$ is the number of terms in the basis, and $a_{i}(x)$ are the coefficients of the basis function. Some examples of commonly used bases are the linear basis

$$
\mathbf{p}^{T}=(1, x) \text { in } 1-\mathrm{D} \text { and } \mathbf{p}^{\mathrm{T}}=(1, x, y) \text { in } 2-\mathrm{D}
$$

and the quadratic basis

$$
\mathbf{p}^{\mathrm{T}}=\left(1, x, x^{2}\right) \text { in } 1-\mathrm{D} \text { and } \mathbf{p}^{\mathrm{T}}=\left(1, x, y, x^{2}, x y, y^{2}\right) \text { in 2-D }
$$

The unknown coefficients $a_{i}(\mathbf{x})$ in (37) can be determined by the minimization of the weighted discrete $L_{2}$ norm

$$
J=\sum_{I}^{n} w\left(\mathbf{x}-\mathbf{x}_{I}\right)\left[\mathbf{P}^{\mathrm{T}}\left(\mathbf{x}_{I}\right) \mathbf{a}\left(\mathbf{x}_{I}\right)-F_{I}\right]^{2}
$$

where $w\left(\mathbf{x}-\mathbf{x}_{I}\right)$ is the weight function with compact support, $n$ is the number of nodes with $w\left(\mathbf{x}-\mathbf{x}_{I}\right)>0$, and is $F_{I}$ the nodal parameter. The minimum of $J$ in Equation (40) with respect to $\mathbf{a}(\mathbf{x})$ leads to set of linear equations

$$
\mathbf{A}(\mathbf{x}) \mathbf{a}(\mathbf{x})=\mathbf{B}(\mathbf{x}) \mathbf{F}
$$

where

$$
\mathbf{A}(\mathbf{x})=\sum_{I=1}^{n} w\left(\mathbf{x}-\mathbf{x}_{I}\right) \mathbf{p}\left(\mathbf{x}_{I}\right) \mathbf{p}^{\mathrm{T}}\left(\mathbf{x}_{I}\right)
$$

and

$$
\mathbf{B}(\mathbf{x})=\left[w\left(\mathbf{x}-\mathbf{x}_{1}\right) \mathbf{p}\left(\mathbf{x}_{1}\right), w\left(\mathbf{x}-\mathbf{x}_{2}\right) \mathbf{p}\left(\mathbf{x}_{2}\right), \ldots, w\left(\mathbf{x}-\mathbf{x}_{n}\right) \mathbf{p}\left(\mathbf{x}_{n}\right)\right]
$$

Thus, the unknown coefficients $\mathbf{a}(\mathbf{x})$ can be obtained from (41) as

$$
\mathbf{a}(\mathbf{x})=\mathbf{A}^{-1}(\mathbf{x}) \mathbf{B}(\mathbf{x}) \mathbf{F}
$$

Substituting (44) into (37), the MLS approximation can be written in standard form as

$$
F^{h}(\mathbf{x})=\sum_{I=1}^{n} N_{I}(\mathbf{x}) F_{I}(\mathbf{x})
$$

where the MLS shape function $N_{I}(\mathbf{x})$ is defined as

$$
N_{I}(\mathbf{x})=\sum_{j=1}^{m} p_{j}(\mathbf{x})\left[\mathbf{A}^{-1}(\mathbf{x}) \mathbf{B}(\mathbf{x})\right]_{j I}=\mathbf{p}^{\mathrm{T}}(\mathbf{x}) \mathbf{A}^{-1}(\mathbf{x}) \mathbf{B}_{I}(\mathbf{x})
$$


with

$$
\mathbf{B}_{I}(\mathbf{x})=w\left(\mathbf{x}-\mathbf{x}_{I}\right) \mathbf{p}\left(\mathbf{x}_{I}\right)
$$

During the MLS approximation, a possible problem is the failure of the method if matrix $\mathbf{A}(\mathbf{x})$ becomes singular. This condition can be avoided by the construction of orthogonal basis functions. An orthogonal basis function set can be formed as [22,23]

$$
\begin{aligned}
& p_{1}=1 \\
& p_{i}=r^{i-1}-\sum_{k=1}^{i-1} \frac{\left(r^{i-1}, p_{k}\right)}{\left(p_{k}, p_{k}\right)} p_{k}, \quad i=2,3, \ldots
\end{aligned}
$$

where the inner product $(f, g)$ is denoted as

$$
(f, g)=\sum_{I=1}^{n} w\left(\mathbf{x}-\mathbf{x}_{I}\right) f\left(\mathbf{x}_{I}\right) g\left(\mathbf{x}_{I}\right)
$$

In this equation, $r$ can be chosen as $\sqrt{x_{1}^{2}+x_{2}^{2}}$ or $x_{1}+x_{2}$ for 2-D problems, and $|x|$ or $x$ for 1-D problems.

In the following, this orthogonal basis function set is used into the MLS approximation, and the weight function is chosen as [23]

$$
w(d)= \begin{cases}\frac{2}{3}-4 d^{2}+4 d^{3}, & d \leqslant \frac{1}{2} \\ \frac{4}{3}+4 d+4 d^{2}-\frac{4}{3} d^{3}, & \frac{1}{2}<d \leqslant 1 \\ 0, & d>1\end{cases}
$$

The compact support domain of the evaluation point is determined by choosing the suitable quantity $R_{0}$ in the formula

$$
d=\left|s-s_{0}\right| / R_{0}
$$

where $s_{0}$ is the local curved co-ordinate of the evaluation point.

\section{BOUNDARY ELEMENT-FREE METHOD}

To carry out the numerical integration, the general boundary and crack surface are firstly separated into a series of sub-domains that are being called 'integral cells' in References [37,38]. Some nodes are then selected on each cell. The choice of cells is arbitrary and independent of the nodes, but each cell must contain enough nodes to ensure that all of the compact support domains cover the crack surface.

The extended displacement density is directly approximated using the MLS approximation of the general boundaries, the extended displacement dislocation density is expressed as (36), and the unknown variable $F_{I}(s)$ is approximated using the MLS approximation.

In our calculation, the source point is chosen as the midpoint between any two adjacent nodes, and a linear equation system can be obtained. To solve the problem, the following single-value 


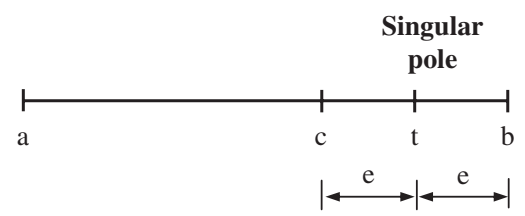

Figure 1. The integral interval is divided into two segments for the evaluation of the singular integrals.

condition of displacement is also discretized on each crack surface:

$$
\int_{\Gamma_{i}} \frac{\partial \Delta u_{I}}{\partial s} \mathrm{~d} s=0
$$

The integral cells are only used so that the integral can be evaluated numerically. The cell is not a boundary element, and neither is a shape function dependent on it. This method is called the boundary element-free method.

When the source point is located inside an integral cell, the kernel of (33) leads to singular integrals of the order $1 / r$. Generally the Cauchy singular integral can be determined analytically or semi-analytically using the BEM, but as the shape functions cannot be known in a closed form, the singular integrals must be evaluated numerically. We find the method of Torino [26] to be very effective for meshless methods, and successfully employed it in our previous work [39]. Some details of the method are described as follows.

As shown in Figure 1, the singular pole $t$ is located on the integral interval from $a$ to $b$ (assuming that point $t$ is nearer to $b$ than to $a$ ). A suitable point $c$ is selected so that point $t$ is equidistant from $c$ and $b$. The $2 m$-point Gauss ruler points are used to draw the segments from $a$ to $c$ and from $c$ to $b$, and the whole integral is then evaluated with the degree of $4 m$ exactness, i.e.

$$
\begin{aligned}
\mathbf{P} \int_{a}^{b} \frac{f(x)}{x-t} \mathrm{~d} x & =\int_{a}^{c} \frac{f(x)}{x-t} \mathrm{~d} t+\mathbf{P} \int_{c}^{b} \frac{f(x)}{x-t} \mathrm{~d} x \\
& =A_{0}^{(1)} f(t)+\sum_{i=1}^{2 m}\left[A_{i}^{(1)} f\left(x_{i}\right)+A_{i}^{(2)} f\left(y_{i}\right)\right]+R_{(2 m)}^{(2)}(f)
\end{aligned}
$$

where

$$
\begin{aligned}
x_{i} & =(c-a) \xi_{i} / 2+(a+c) / 2, \quad y_{i}=(b-c) \xi_{i} / 2+(b+c) / 2 \\
A_{i}^{(1)} & =(c-a) H_{i} / 2\left(x_{i}-t\right), \quad A_{i}^{(2)}=(b-c) H_{i} / 2\left(y_{i}-t\right) \\
A_{0}^{(1)} & =\ln \left|\frac{c-t}{a-t}\right|-\sum_{i=1}^{2 m} A_{i}^{(1)}
\end{aligned}
$$

with $\xi_{i}$ and $H_{i}$ being the Gauss quadrature point and weight, respectively. 
Around the crack tip, a relation between the extended crack opening displacements and the extended SIFs is obtained [4] as

$$
\Delta \mathbf{u}(r)=2 \sqrt{\frac{2 r}{\pi}} \operatorname{Re}(\mathbf{Y}) \mathbf{K}
$$

where matrix $\mathbf{Y}$ is defined by (19) and $\mathbf{K}$ is the extended SIF vector that is defined as

$$
\mathbf{K}=\left\{K_{\mathrm{II}}, K_{\mathrm{I}}, K_{\mathrm{IV}}\right\}^{\mathrm{T}}
$$

where $K_{\mathrm{I}}$ and $K_{\mathrm{II}}$ are the usual SIFs and $K_{\mathrm{IV}}$ is the electric displacement intensity factor.

By combining (55) and (36), the extended SIFs can be evaluated from the extended displacement dislocation density by

$$
\mathbf{K}=\sqrt{\frac{\pi}{2\left|s_{1}-s_{2}\right|}}[\operatorname{Re}(\mathbf{Y})]^{-1} \frac{\partial \Delta \mathbf{u}(r)}{\partial s}
$$

\section{NUMERICAL EXAMPLES}

Four types of example problems are carefully chosen to demonstrate the accuracy and efficiency of the proposed method. In all computations, the cells and nodes are uniformly chosen and the scale $R_{0}$ is maintained at three times the distance between the two adjacent nodes to ensure that six nodes are involved for the ordinary evaluation points.

\subsection{Straight crack in an unbounded domain}

The first example is an infinite PZT-4 plate [20] that contains a horizontal straight crack along the $x_{1}$-axis. The crack length is $a$, and the origin of the Cartesian co-ordinate system oxy is located at the centre of the crack. The plate is under a uniform far-field stress or electric displacement that is applied in the $x_{2}$-direction. The material constants are given in Table I, and the axis of symmetry of the material is the $x_{2}$-axis.

The proposed method shows a similar trend of convergence as that in Reference [39]. For this example, we have used 15 integral cells and chosen 46 nodes on the crack surface. The numerical

\begin{tabular}{|c|c|c|c|c|}
\hline \multicolumn{5}{|c|}{ Material constants for examples $1-3$} \\
\hline $\begin{array}{l}C_{11}(\mathrm{GPa}) \\
139\end{array}$ & $C_{12}(\mathrm{GPa})$ & $C_{22}(\mathrm{GPa})$ & $C_{66}(\mathrm{GPa})$ & \\
\hline $\begin{array}{l}e_{21}\left(\mathrm{C} / \mathrm{m}^{2}\right) \\
-5.2\end{array}$ & $\begin{array}{c}e_{22}\left(\mathrm{C} / \mathrm{m}^{2}\right) \\
15.1\end{array}$ & $\begin{array}{c}e_{16}\left(\mathrm{C} / \mathrm{m}^{2}\right) \\
12.7\end{array}$ & $\begin{array}{c}\varepsilon_{11}(\mathrm{C} /(\mathrm{GV} \mathrm{m})) \\
6.461\end{array}$ & $\begin{array}{c}\varepsilon_{22}(\mathrm{C} /(\mathrm{GV} \mathrm{m})) \\
5.62\end{array}$ \\
\hline $\begin{array}{l}\text { Material con } \\
C_{11}(\mathrm{GPa}) \\
139\end{array}$ & $\begin{array}{c}\text { nts for exampl } \\
C_{12}(\mathrm{GPa}) \\
74.3\end{array}$ & $\begin{array}{c}C_{22}(\mathrm{GPa}) \\
113\end{array}$ & $\begin{array}{c}C_{66}(\mathrm{GPa}) \\
25.6\end{array}$ & \\
\hline $\begin{array}{l}e_{21}\left(\mathrm{C} / \mathrm{m}^{2}\right) \\
-6.98\end{array}$ & $\begin{array}{c}e_{22}\left(\mathrm{C} / \mathrm{m}^{2}\right) \\
13.84\end{array}$ & $\begin{array}{c}e_{16}\left(\mathrm{C} / \mathrm{m}^{2}\right) \\
13.44\end{array}$ & $\begin{array}{c}\varepsilon_{11}(\mathrm{C} /(\mathrm{GV} \mathrm{m})) \\
6.0\end{array}$ & $\begin{array}{c}\varepsilon_{22}(\mathrm{C} /(\mathrm{GV} \mathrm{m})) \\
5.47\end{array}$ \\
\hline
\end{tabular}
results for $F_{2}(x)$ and $F_{3}(x)\left(F_{1}(x)=0\right)$ are shown in Figures 2 and 3 for a uniform far-field

Table I. Material constants. 


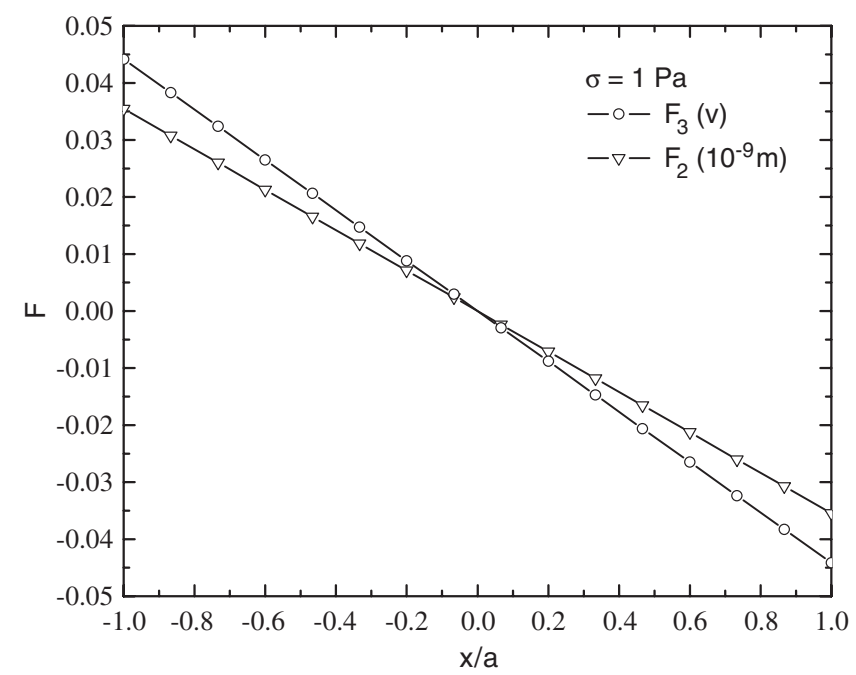

Figure 2. The obtained $F_{2}(x)$ and $F_{3}(x)$ versus $x$ for a straight crack in an infinite PZT-4 plate under uniform far-field tension.

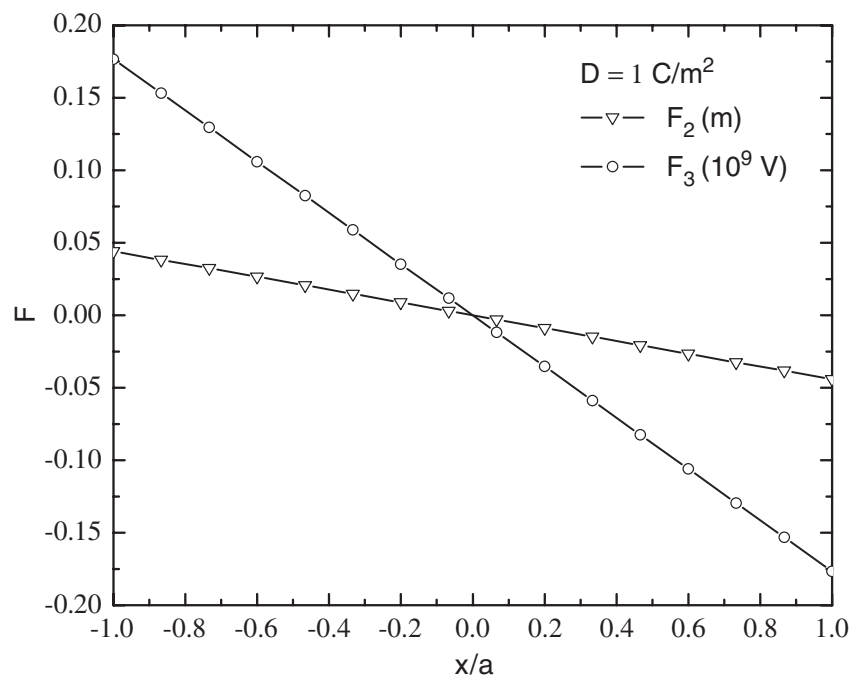

Figure 3. The obtained $F_{2}(x)$ and $F_{3}(x)$ versus $x$ for a straight crack in an infinite PZT-4 plate under uniform far-field electric displacement.

tension and electric displacement. $F_{I}(x)$ is actually a linear function [2,4], and our computation shows that very accurate results are obtained by using the MLS approximation. Here, $F_{1}(x)$ and $F_{2}(x)$ are in unit $m$ and $F_{3}(x)$ is $V$. The extended SIFs, which are directly evaluated from the extended dislocation densities by (57), are listed in Table II along with the exact SIFs [2,4]. It 
Table II. Normalized extended stress intensity factors for a horizontal straight crack in an infinite piezoelectric plate.

\begin{tabular}{lccc}
\hline & $K_{\mathrm{I}} / \sigma_{22} \sqrt{\pi a}$ & $K_{\mathrm{II}} / \sigma_{12} \sqrt{\pi a}$ & $K_{\mathrm{IV}} / D \sqrt{\pi a}$ \\
\hline Numerical & 0.99997 & 0.99997 & 0.99997 \\
Exact & 1 & 1 & 1 \\
\hline
\end{tabular}

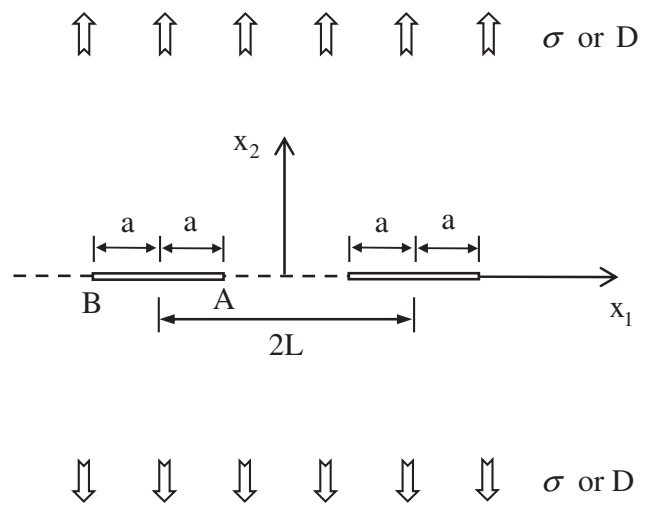

Figure 4. Two collinear cracks in an unbounded piezoelectric medium.

should be pointed out that the extended SIFs are uncoupled for the straight crack, which means that a far-field stress does not induce the electric displacement intensity factor and a far-field electric displacement does not induce the SIF. Moreover, in order to obtain the extended displacement, we have integrated the corresponding density terms along the boundary or crack surface. This additional computation work is unavoidable in our proposed method of solution.

\subsection{Two collinear cracks in an unbounded domain}

This example studies the interaction of two collinear cracks of the same length in an infinite piezoelectric solid (see Figure 4). The same material constants as those in Section 7.1 are used. The piezoelectric solid is subjected to a uniform far-field stress or electric displacement. From our computation, it shows that the extended SIFs are still uncoupled, and $\sigma_{22}, \sigma_{12}$, and $D_{2}$ only induce the mode I and mode II SIFs and the electric displacement intensity factor, respectively. Thus, the normalized extended SIFs should have the same quantities as those in the electromechanical uncoupled cases. The results are listed in Table III along with the exact solutions for two collinear cracks in an unbounded elastic solid [40]. These results are close to the exact solutions, even when the two cracks are placed very near to each other, which reveal that the MLS approximation has a good ability to model the unknown variable.

\subsection{Inclined crack in a rectangular plate}

The third example corresponds to a rectangular PZT-4 solid contains a central and straight cracks with $45^{\circ}$ inclined in the horizontal direction (Figure 5). A uniform tension or electric displacement 
Table III. Normalized extended stress intensity factors for two collinear cracks in an infinite piezoelectric plate $\left(Y_{\mathrm{I}}=K_{\mathrm{I}} / \sigma_{22} \sqrt{\pi a}, Y_{\mathrm{II}}=K_{\mathrm{II}} / \sigma_{12} \sqrt{\pi a}, Y_{\mathrm{IV}}=K_{\mathrm{IV}} / D \sqrt{\pi a}\right)$.

\begin{tabular}{|c|c|c|c|c|}
\hline \multirow[b]{2}{*}{$a / L$} & \multicolumn{2}{|c|}{$Y_{\mathrm{IA}}\left(=Y_{\mathrm{IIA}}=Y_{\mathrm{IVA}}\right)$} & \multicolumn{2}{|c|}{$Y_{\mathrm{IB}}\left(=Y_{\mathrm{IIB}}=Y_{\mathrm{IVB}}\right)$} \\
\hline & Present & Exact* & Present & Exact* \\
\hline 0.1 & 1.00130 & 1.00132 & 1.00119 & 1.00120 \\
\hline 0.2 & 1.00564 & 1.00566 & 1.00462 & 1.00462 \\
\hline 0.3 & 1.01381 & 1.01383 & 1.01016 & 1.01017 \\
\hline 0.4 & 1.02715 & 1.02717 & 1.01786 & 1.01787 \\
\hline 0.5 & 1.04794 & 1.04796 & 1.02795 & 1.02795 \\
\hline 0.6 & 1.08038 & 1.08040 & 1.04093 & 1.04094 \\
\hline 0.7 & 1.13324 & 1.13326 & 1.05786 & 1.05786 \\
\hline 0.8 & 1.22890 & 1.22893 & 1.08106 & 1.08107 \\
\hline 0.9 & 1.45377 & 1.45387 & 1.11741 & 1.11741 \\
\hline
\end{tabular}

*Exact solutions for two collinear cracks in an unbounded elastic solid [38].

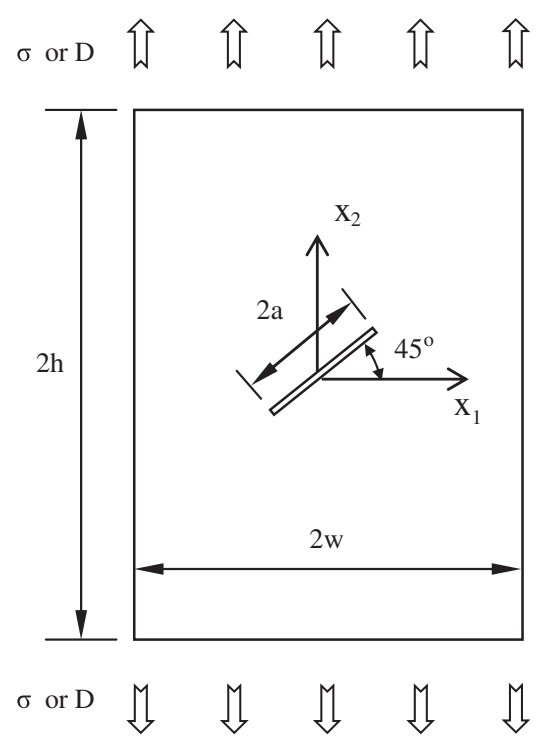

Figure 5. Inclined crack in a rectangular piezoelectric plate.

is applied in the $x_{2}$-direction. The material constants are the same as those used in Section 7.1. For comparison purpose, the ratios of crack length to width and height to width are taken as $a / w=0.2$ and $h / w=2$, respectively [20,21]. The single-side MLS approximation [37,38] is used for the evaluation points that are close to the corner of the outside general boundary. Both electromechanical coupled and uncoupled $\left(\mathrm{e}_{i j k}=0\right)$ cases are computed. The normalized extended SIFs for two loading conditions are listed in Tables IV and V. A good agreement with those of Pan [20] is evident. In Table IV, $D^{*}$ is a nominal electric displacement in units of $\mathrm{cm}^{-2}$, with an amplitude that is equal to that $\sigma_{22}$ of in units of $\mathrm{Nm}^{-2}$. In Table $\mathrm{V}, \sigma^{*}$ is a nominal stress in units of $\mathrm{Nm}^{-2}$ with an amplitude that is equal to that of $D_{2}$ in units of $\mathrm{cm}^{-2}$. 
Table IV. Normalized extended stress intensity factors for an inclined crack in a rectangular piezoelectric solid loaded by normal stress.

\begin{tabular}{lcccc}
\hline & & $K_{\mathrm{I}} / \sigma \sqrt{\pi a}$ & $K_{\mathrm{II}} / \sigma \sqrt{\pi a}$ & $K_{\mathrm{IV}} / D^{*} \sqrt{\pi a}$ \\
\hline Coupled & Present & 0.52191 & 0.50719 & $-2.95177 \times 10^{-12}$ \\
& Pan [20] & 0.5303 & 0.5151 & $-2.97 \times 10^{-12}$ \\
Uncoupled & Present & 0.51974 & 0.50706 & 0.0000 \\
& Pan [20] & 0.5275 & 0.5151 & 0.0000 \\
\hline
\end{tabular}

Table V. Normalized extended stress intensity factors for an inclined crack in a rectangular piezoelectric solid loaded by electric displacement.

\begin{tabular}{lccll}
\hline & & $K_{\mathrm{IV}} / D \sqrt{\pi a}$ & $K_{\mathrm{I}} / \sigma^{*} \sqrt{\pi a}$ & $K_{\mathrm{II}} / \sigma^{*} \sqrt{\pi a}$ \\
\hline Coupled & Present & -0.71654 & $-1.37793 \times 10^{6}$ & $1.63115 \times 10^{6}$ \\
& Pan [20] & -0.72785 & $-1.417 \times 10^{6}$ & $1.692 \times 10^{6}$ \\
\multirow{2}{*}{ Uncoupled } & Present & -0.71651 & 0.0000 & 0.0000 \\
& Pan [20] & -0.72776 & 0.0000 & 0.0000 \\
\hline
\end{tabular}

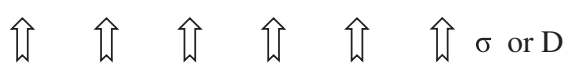

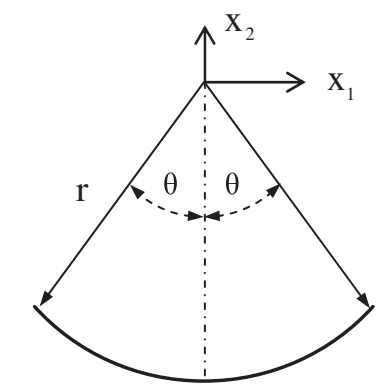

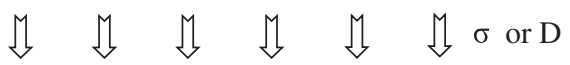

Figure 6. Circular-arc crack in an unbounded piezoelectric medium.

\subsection{Circular-arc crack in an unbounded domain}

To test the proposed method for treating curved cracks, a circular-arc crack in an infinite anisotropic piezoelectric plate (Figure 6) is selected. The crack region is subjected to a uniform far-field tension or electric displacement in the $x_{2}$-direction. The material constants of this problem have been given earlier in Table I. The material considered is symmetric about the $x_{2}$-axis. To furnish the solution, we have used 30 integral cells and 90 nodes in the numerical computation, and the semi-angle $\theta$ 


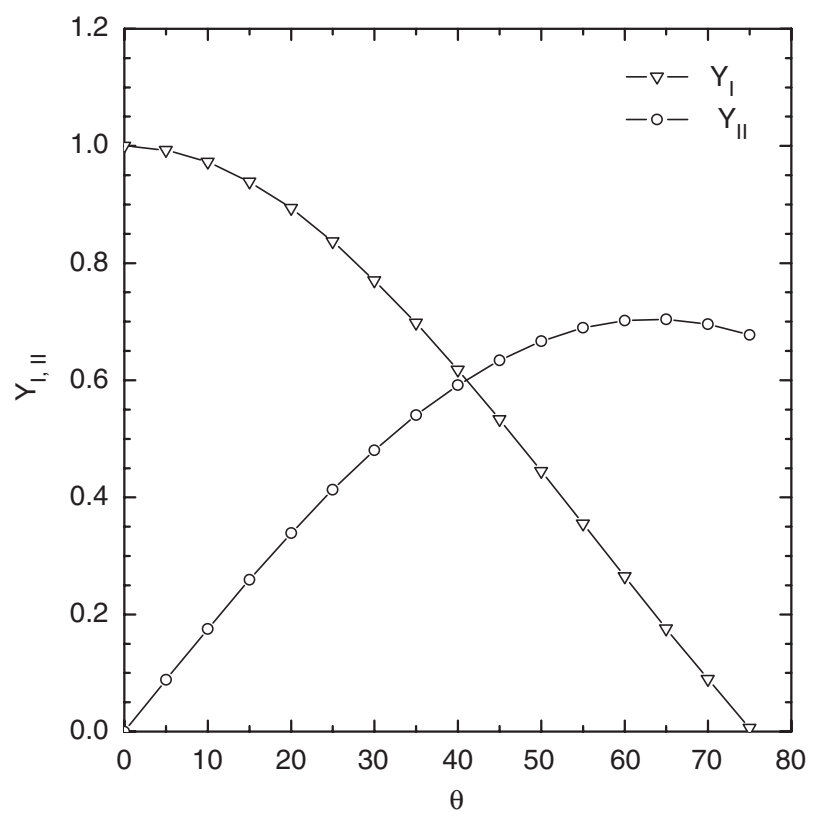

Figure 7. Dimensionless mode I an II stress intensity factors $\mathrm{Y}_{\mathrm{I}, \mathrm{II}}=K_{\mathrm{I}, \mathrm{II}} / \sigma \sqrt{\pi r \sin \theta}$ versus $\theta$.

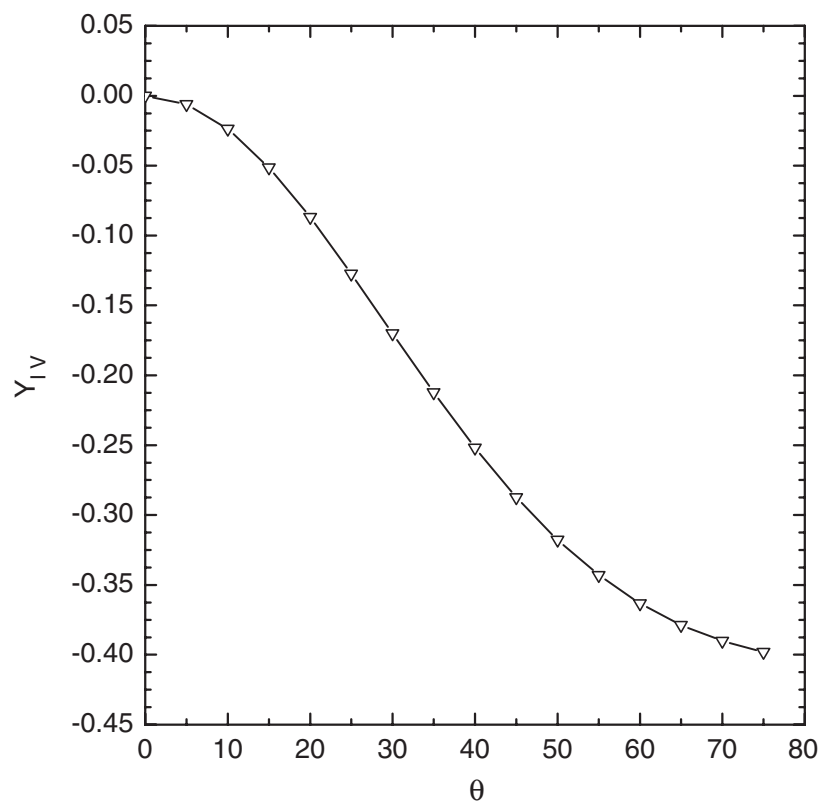

Figure 8. Dimensionless electric displacement intensity factors $\mathrm{Y}_{\mathrm{IV}}=K_{\mathrm{IV}} / \sigma \chi \sqrt{\pi r \sin \theta}$ versus $\theta$. 


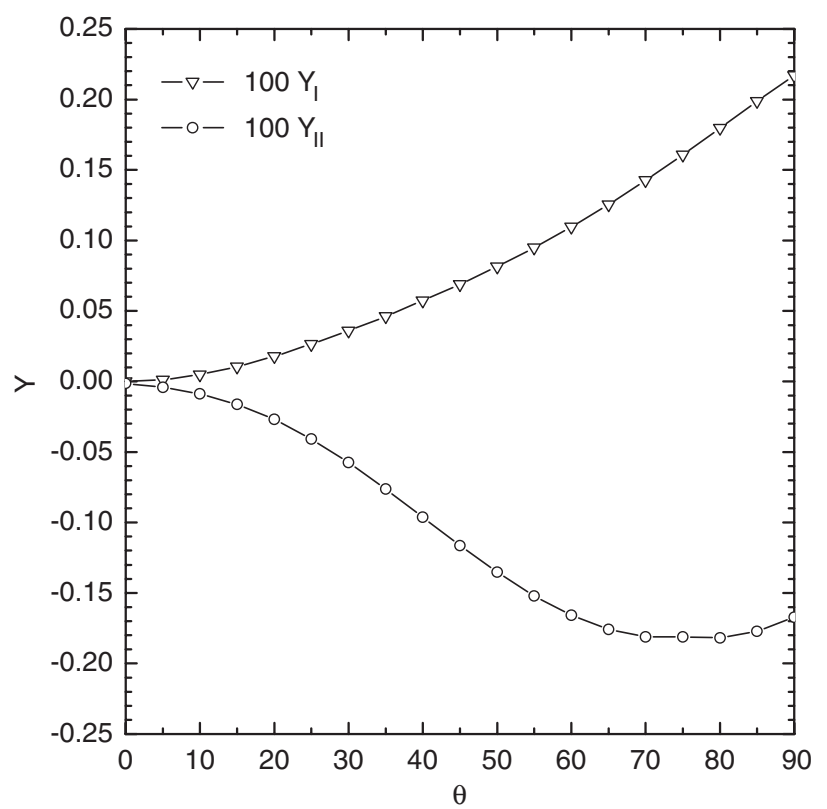

Figure 9. Dimensionless mode I and II stress intensity factors $\mathrm{Y}_{\mathrm{I}, \mathrm{II}}=K_{\mathrm{I}, \mathrm{II}} \chi / D \sqrt{\pi r \sin \theta}$ versus $\theta$.

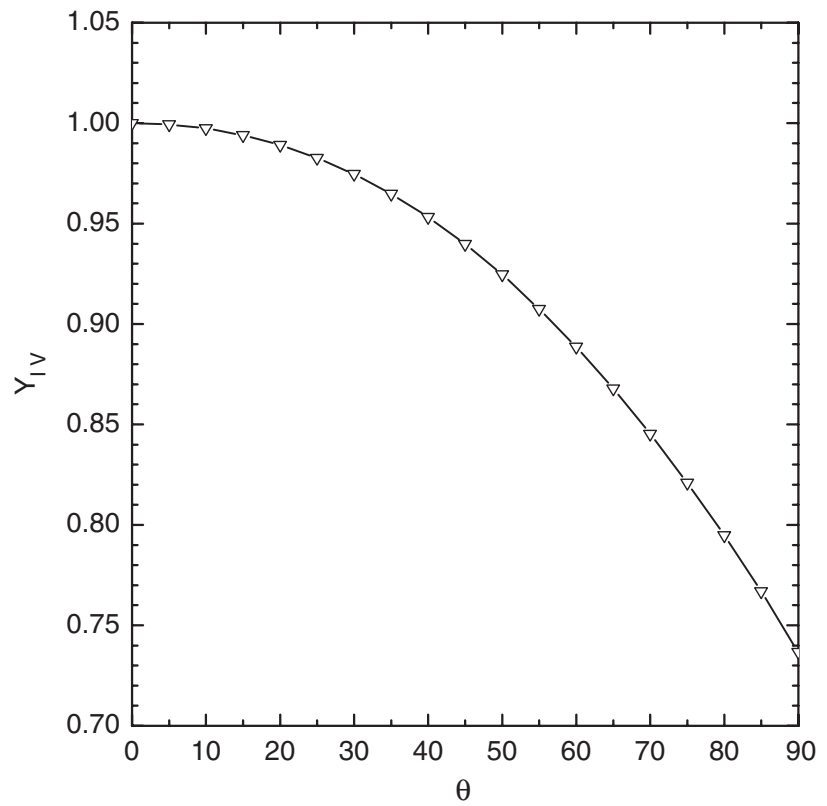

Figure 10. Dimensionless electric displacement intensity factors $\mathrm{Y}_{\mathrm{IV}}=K_{\mathrm{IV}} / D \sqrt{\pi r \sin \theta}$ versus $\theta$. 
changes from $0^{\circ}$ to $75^{\circ}$ or $90^{\circ}$. The ratio $\chi$ between the dielectric constant $\varepsilon_{22}$ and the piezoelectric constant $e_{22}$ is introduced here to present a dimensionless electric displacement density factor for the far-field tension and a stress density factor for the far-field electric displacement. The dimensionless extended SIFs are plotted as the function of $\theta$ in Figures 7-10. This problem was recently studied by Garcia et al. [21], and the present results show a good agreement with those reported by Garcia et al. [21].

\section{CONCLUSIONS}

An extended traction BIE is presented for the fracture analysis of 2-D piezoelectric solids using integration by parts, in which the kernels of the new integral equation only involve a singularity of order $1 / r$. The equation can be directly applied to both the general boundary and the crack surfaces of anisotropic piezoelectric solids. The boundary conditions on the crack surface are incorporated, and the upper and lower surfaces do not have to be considered separately. The basic unknowns are the tangential derivative of the extended displacement and the extended crack opening displacement, and the equation is very effective for the analysis of problems with straight and curved cracks. The extended displacement dislocation densities on the crack surface are expressed as the product of the characteristic terms and unknown weight functions. The unknown weight functions and extended displacement densities on the outside boundary are approximated by the MLS method. A boundary element-free method is established, and good results are obtained for the considered examples. This study has also shown that the MLS approximation is a flexible interpolation method.

\section{ACKNOWLEDGEMENTS}

The work described in this paper was fully supported by the City University of Hong Kong Strategic Research Grant [Project No. 7001961].

\section{REFERENCES}

1. Barnett DM, Lothe J. Dislocations and line charges in anisotropic piezoelectric insulators. Physica Status Solidi B-Basic Research 1975; 67:105-611.

2. Pak YE. Linear electric-elastic fracture mechanics of piezoelectric materials. International Journal of Fracture 1992; 54:79-110.

3. Sosa H. On fracture mechanics of piezoelectric solids. International Journal of Solids and Structures 1992; 29:2613-2622.

4. Suo Z, Kuo CM, Barnett DM. Fracture mechanics for piezoelectric ceramics. Journal of the Mechanics and Physics of Solids 1992; 40:739-765.

5. Chau KT, Wang YB. A new boundary integral formulation for plane elastic bodies containing cracks and holes. International Journal of Solids and Structures 1999; 36:2041-2074.

6. Wang YB, Chau KT. A new boundary element for plane elastic problems involving cracks and holes. International Journal of Fracture 1997; 89:1-20.

7. Qin TY, Tang RJ. Finite-part integral and boundary element method to solve embedded planar crack problems. International Journal of Fracture 1993; 60:373-381.

8. Rangelov T, Dineva P, Gross D. A hyper-singular traction boundary integral equation method for stress intensity factor computation in a finite cracked body. Engineering Analysis with Boundary Elements 2003; 27:9-21.

9. Hong HK, Chen JT. Derivations of integral-equations of elasticity. Journal of Engineering Mechanics (ASCE) 1988; 114:1028-1044. 
10. Portela A, Aliabadi MH, Rooke DP. The dual boundary element method: effective implementation for crack problems. International Journal for Numerical Methods in Engineering 1992; 33:1269-1287.

11. Bui HD. Integral-equations method for solving the problem of a plane crack of arbitrary shape. Journal of the Mechanics and Physics of Solids 1977; 25:29-39.

12. Richardson JD, Cruse TA. Weakly singular stress-BEM for 2D elastostatics. International Journal for Numerical Methods in Engineering 1999; 45:13-35.

13. Li S, Mear ME, Xiao L. Symmetric weak-form integral equation method for three-dimensional fracture analysis. Computer Methods in Applied Mechanics and Engineering 1998; 151:435-459.

14. Liang YC, Hwu C. Electromechanical analysis of defects in piezoelectric materials. Smart Materials and Structures 1996; 5:314-320.

15. Ding HJ, Wang GQ, Chen WQ. A boundary integral formulation and 2D fundamental solutions for piezoelectric media. Computer Methods in Applied Mechanics and Engineering 1998; 158:65-80.

16. Liu YL, Fan H. Analysis of thin piezoelectric solids by the boundary element method. Computer Methods in Applied Mechanics and Engineering 2002; 191:2297-2315.

17. Rajapakse RKND, Xu XL. Boundary element modeling of cracks in piezoelectric solids. Engineering Analysis with Boundary Elements 2001; 25:771-781.

18. Denda M, Lua J. Development of the boundary element method for 2D piezoelectricity. Composites: Part B 1999; 30:699-707.

19. Davi G, Milazzo A. Multi-domain boundary integral formulation for piezoelectric materials fracture mechanics. International Journal of Solids and Structures 2001; 38:7065-7078.

20. Pan E. A BEM analysis of fracture mechanics in 2D anisotropic piezoelectric solids. Engineering Analysis with Boundary Elements 1999; 23:67-76.

21. Garcia F, Saez A, Dominguez J. Anisotropic and piezoelectric materials fracture analysis by BEM. Computers and Structures 2005; 83:804-820.

22. Lu YY, Belytschko T, Gu L. A new implementation of the element free Galerkin method. Computer Methods in Applied Mechanics and Engineering 1994; 113:397-414.

23. Kitipornchai S, Liew KM, Cheng YM. A boundary element-free method (BEFM) for three dimensional elasticity problems. Computational Mechanics 2005; 36:13-20.

24. Liew KM, Cheng YM, Kitipornchai S. Boundary element-free method (BEFM) for two-dimensional elastodynamic analysis using Laplace transform. International Journal for Numerical Methods in Engineering 2005; 64(12): $1610-1627$.

25. Liew KM, Cheng YM, Kitipornchai S. Boundary element-free method (BEFM) and its application to twodimensional elasticity problems. International Journal for Numerical Methods in Engineering 2006; 65(8): 1310-1332.

26. Torino GM. The numerical evaluation of one-dimensional Cauchy principal value integrals. Computing 1982; 29:337-354.

27. Pan E, Tonon F. Three-dimensional Green's functions in anisotropic piezoelectric solids. International Journal of Solids and Structures 2000; 37:943-958.

28. Sun YZ, Yang SS, Wang YB. A new formulation of boundary element method for cracked anisotropic bodies under anti-plane shear. Computer Methods in Applied Mechanics and Engineering 2003; 192:2633-2648.

29. Wang YB, Sun YZ. A new boundary integral equation method for cracked 2-D anisotropic bodies. Engineering Fracture Mechanics 2005; 72:2128-2143.

30. Nisitani H. Mechanics of Fracture 5, Sih CG (ed.). Noordhoff International Publishing: Leyden, 1978.

31. Noda NA, Oda K. Numerical solutions of singular integral equations in the crack analysis using the body force method. International Journal of Fracture 1992; 58:285-304.

32. Comninou M. The interface crack. Journal of Applied Mechanics 1997; 44:631-636.

33. Noda NA, Matsuo T. Numerical solutions of singular integral-equations having Cauchy-type singular kernel by means of expansion method. International Journal of Fracture 1993; 63:229-245.

34. Noda NA, Oda K. Effect of curvature at the crack tip on the stress intensity factor for curved cracks. International Journal of Fracture 1993; 64:239-249.

35. Wang XD, Meguid SA, Papanikos P. Analysis of curved cracks emanating from adjacent holes. Engineering Fracture Mechanics 1999; 64:337-355.

36. Belytschko T, Krongauz Y, Organ D, Fleming M, Krysl P. Meshless methods: an overview and recent developments. Computer Methods in Applied Mechanics and Engineering 1996; 139:3-47. 
37. Mukherjee YX, Mukherjee S. The boundary node method for potential problems. International Journal for Numerical Methods in Engineering 1997; 40:797-815.

38. Kothnur VS, Mukherjee S, Mukherjee YX. Two-dimensional linear elasticity by the boundary node method. International Journal of Solids and Structures 1999; 36:1129-1147.

39. Sun YZ, Zhang Z, Kitipornchai S, Liew KM. Analyzing the interaction between collinear interfacial cracks by an efficient boundary element-free method. International Journal of Engineering Science 2006; 44(1-2):37-48.

40. Tada H, Pair PC, Irwin GR. The Stress Analysis of Cracks Handbook (3rd edn). Professional Engineering Publishing: London, 2000. 